\title{
Padrões de Interfaces Sociais no Desenho do BOA
}

\section{Social Interface Patterns for the Design of BOA System}

\begin{abstract}
Resumo: A existência de comunidades online em torno de objectos sociais e cada vez mais habitual na Internet, tendo algumas enorme popularidade. Este trabalho enquadra-se no âmbito de comunidades online na área do ensino $e$ aprendizagem, ao propor o sistema BOA (Bolsa de Objectos de Aprendizagem) como uma plataforma de Objectos de Aprendizagem (OA) versátil e configurável, que introduz conceitos inovadores para promover a colaboração entre utilizadores, privilegiando uma competitividade saudável $e$ consequente qualidade dos OA submetidos. A plataforma BOA tem sido desenvolvida e aplicada em cenários concretos ao longo dos últimos anos, suportando diferentes tipos de comunidades, tais como o VemAprender, net ou o BOA-GPI. Este artigo apresenta o resultado da análise e avaliação da plataforma BOA com base no referencial da computação social perspectivando, consequentemente, um conjunto de aspectos relevantes a desenvolver. Em particular, é analisado e avaliado o BOA contra um conjunto de padrões de interfaces sociais (i.e., Profile, PersonnalDashboard Favorites, Displaying, Send/Share widget, Embbeding, Send/Receive Invitations) que são manifestos em sistemas como outube, SlideShare ou o Flickr. Por fim, e com base nesta avaliaça, são discutidos os aspectos relevantes a experincia paratilizão un un experiencia de utizaça superior e potenciar a respectiva comuño avaliação do BOA, proposta neste artigo, poderá ser adoptada Palavras-chave: Objectos de Aprendizagem. Comunidades Online. Padrões de Interfaces Sociais. Objectos Sociais.
\end{abstract}

Abstract: Online communities based on social objects are increasingly popular in the Internet, some of them with huge popularity. The scope of this research is in the area of online communities, particularly those supporting e-learning based scenarios. We consider the BOA ("Bolsa de Objectos de Aprendizagem" in Portuguese, or "Learning Objects Pool") as a versatile and configurable Web-based platform and a learning objects repository, which introduces innovative concepts to promote collaboration between users and also the quality of the submitted learning objects. The BOA platform has been developed and applied to different scenarios over the recent years, supporting different types of communities, such as BOA-GPI or VemAprender.net. This paper presents the results of the analysis and evaluation of $\mathrm{BOA}$ platform that is conducted according the social computing framework, therefore, foreseeing a number of relevant aspects for its future development. In particular, the BOA platform is reviewed and evaluated against a set of social interface patterns (i.e., Profile, PersonnalDashboard, Favorites, Displaying, Send/Share widget, Embbeding, Send/Receive Invitations) that are presented in systems such as Youtube Slideshare or Flickr. Finally, and based on such review, we discuss relevant aspects to be considered to provide BOA's end-users superior experience and enhance their community We believe that the analysis and evaluation approach proposed in this paper may be adopted with advantage to assess any other related platform or online community. Keywords: Interface Patterns. Social Objects.

SILVA, Alberto Rodrigues da. Padrões de Interfaces Sociais no Desenho do BOA. Informática na Educação: teoria \& prática, Porto Alegre, v. 14, n. 1, p. 167-181, jan./jun. 2011.

\section{Alberto Rodrigues da Silva}

Universidade Técnica de Lisboa

\section{Introdução}

$\mathrm{O}$ ensino e a aprendizagem têm sofrido transformações na sua forma e método ao tirarem partido das potencialidades da Internet. A popularidade da Internet originou um aumento dos modelos e das tecnologias de e-learning, o que tem provocado um aumento de divulgação, de produção e mesmo de comercialização de objectos de aprendizagem (DOWNES, 2004). Um OA (objecto de aprendizagem) é qualquer recurso digital ou não digital, suportado por meios tecnológicos, que possa ser usado, reutilizado ou referenciado em processos de ensino-aprendizagem (DOORTON, 2004), (MCGREAL, 2004).

Os repositórios de objectos de aprendizagem são plataformas que armazenam recursos educativos $e$ respectivos metadados (ou apenas os metadados), providenciando diferentes funcionalidades, em particular mecanismos de pesquisa e exploração desses recursos. É neste âmbito que surgiu a plataforma BOA (SILVA; SILVA, 2006), (CARLOTA; SILVA; DINIS, 2009), (DINIS; SILVA, 2009) que, em relação a outros sistemas do género tais como ARIADNE (www.ariadne-eu.org), EdNA (edna.edu.au), MERLOT (www.merlot.org), Wisc-Online (www.wisc-online.com) ou o e-Escola (www.e-escola.pt), introduz aspectos inovadores, designadamente a metáfora de "bolsa de valores" em que se baseia. Esta metáfora é suportada por um mecanismo de créditos (ou pontos) que permite simultaneamente avaliar o nível de 
colaboração dos utilizadores assim como o valor dos OA. Esta valoração é definida continuamente como reflexo do interesse dos utilizadores pelos OA em questão.

O trabalho discutido neste artigo parte das conclusões do artigo "Casos de Estudo com a Bolsa de Objectos de Aprendizagem: Análise na Perspectiva da Computação Social" (SILVA; DINIS, 2010), a partir das quais percebemos que a comunidade criada à volta da plataforma BOA apresenta limitações que podem e devem ser ultrapassadas, para que os níveis de partilha, divulgação e participação no seio destas comunidades sejam incrementados. Depreendem-se também alguns dos actuais aspectos da plataforma, quer em termos sociais quer em termos tecnológicos. Em termos sociais, o trabalho aponta para que seja feita uma maior divulgação da plataforma, de modo a serem atenuados alguns preconceitos de preocupações dos utilizadores, relacionados com temas como direitos de autor e disponibilização pública de trabalhos pessoais. Em termos tecnológicos, são sugeridas novas funcionalidades que poderão ser implementadas na plataforma, de forma a tornar a comunidade BOA mais dinâmica, através da participação de mais utilizadores.

Considerando como referencial para a análise do desenho de interfaces sociais o trabalho realizado por Crumlish e Malone (2009), foram identificados os seguintes padrões que irão ser utilizados neste trabalho, como referencial de comparação, de forma a suportar uma evolução qualitativa da plataforma BOA, designadamente os padrões: PROFILE, PERSONAL DASHBOARD, FAVORITES; DISPLAYING; SEND/SHARE WIDGET E EMBEDDING; e INVITATIONS.

Este artigo tem como principal objectivo analisar e discutir soluções para que a plataforma BOA ultrapasse ou mitigue as questões referidas. Recorremos para tal, à análise de um conjunto de comunidades online que apresentam um grande sucesso junto dos seus utilizadores, nomeadamente o Youtube, SlideShare e Flickr que, embora não tendo preocupações particulares de carácter educacional, como as plataformas referidas, constituem referências destacadas na área da computação social.

O artigo encontra-se organizado em seis secções. A Secção 2 introduz genericamente a plataforma BOA, os seus conceitos e aspectos distintivos. A Secção 3 introduz os padrões de desenho de interfaces sociais que são considerados com maior relevância no artigo. A secção 4 analisa e discute, com base nos padrões referidos, três comunidades online de destaque: Youtube, SlideShare e Flickr. A Secção 5 analisa e discute esses mesmos padrões no âmbito da plataforma BOA, concluindo-se, na Secção 6 , as principais motivações para o trabalho de investigação em curso.

\section{Sistema BOA}

O Sistema BOA (Bolsa de Objectos de Aprendizagem) é uma plataforma Web que funciona como um repositório de objectos de aprendizagem e que promove a partilha e colaboração entre os seus utilizadores.

O BOA é uma plataforma que pode apresentar diferentes instâncias. Por exemplo, as Figuras 1 e 2 apresentam, respectivamente, écrãs das páginas iniciais das instâncias BOA-GPI (em isg.inescid.pt/BOA-GPI/) e VemAprender (em www.vemaprender.net).

Apresentam-se de seguida os principais conceitos subjacentes à plataforma BOA que podem ser consultados com maior detalhe nos artigos "The Learning Object Pool and the BOA-GPI Case Study" (CARLOTA; SILVA; DINIS, 2009) e "Application Scenarios for the Learning Objects Pool" (DINIS; SILVA, 2009).

\subsection{Principais Conceitos}

Os conceitos principais subjacentes à plataforma BOA são sugeridos na Figura 3 . Um Grupo agrega logicamente um conjunto de Utilizadores e de OA devidamente categorizados segundo uma hierarquia de Tópicos (definida ao nível de cada Grupo).

O OA é o elemento central da plataforma e agrega a informação submetida pelo respectivo autor: (1) o conteúdo propriamente dito, i.e. um ficheiro segundo um formato aceite pelo sistema (e.g. PDF, 
DOC, PPT); (2) os respectivos metadados que o descrevem; (3) informação complementar, submetida pelos utilizadores que o adquiriram previamente, tais como informação relativa a classificação do OA, comentários, sugestões de melhoria, experiências educativas, ou boas práticas de utilização; ou ainda (4) informação submetida pelos seus revisores.

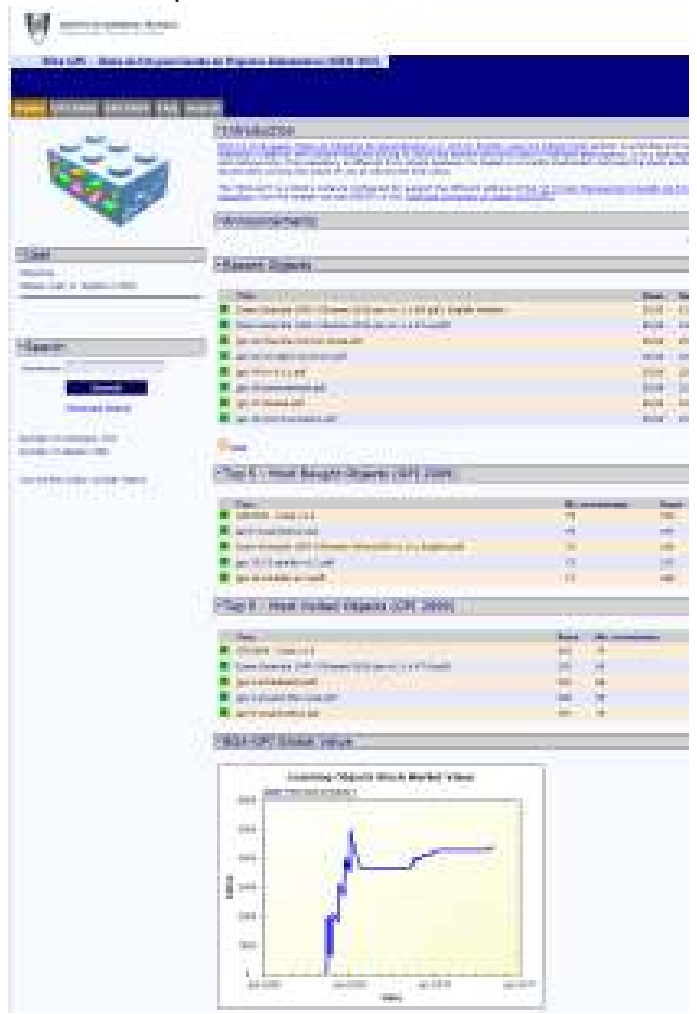

Figura 1 - Página inicial da instância BOA-GPI

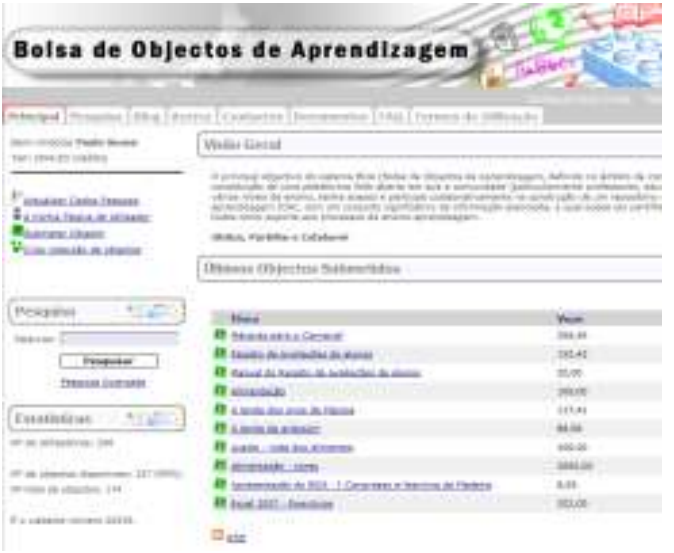

Figura 2 - Página inicial da instância VemAprender.net
Os metadados seguem o Dublin Core (dublincore.org) com algumas extensões que completam a informação dos OA, e.g., o valor inicial e o valor mínimo aceite para venda do OA.

Por outro lado, o conceito de Grupo traduz adequadamente a noção de "comunidade", e permite suportar sobre uma mesma instância do BOA diferentes grupos com regras distintas e mecanismos de configuração, gestão e utilização independentes entre si.

A cada Grupo são associados Utilizadores, podendo-Ihes atribuir distintos papéis, designadamente: (1) consultor, que permite pesquisar, pesquisar e comprar OA desse grupo; (2) membro, que adicionalmente, permite submeter OA nesse grupo; (3) revisor, que permite rever OA submetidos; e (4) gestor, que permite gerir os vários aspectos funcionais do grupo.

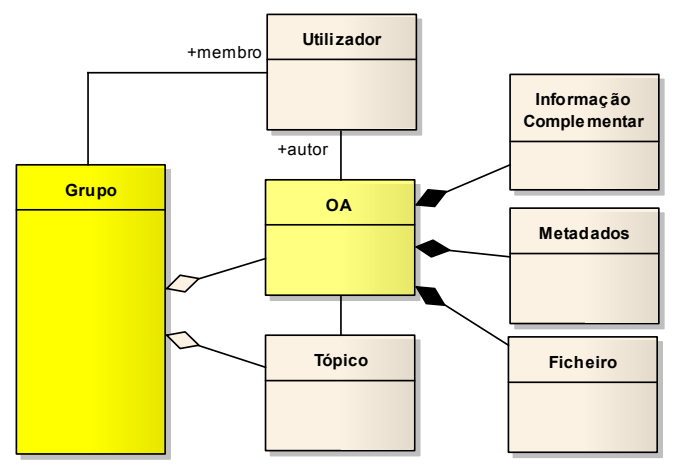

Figura 3 - Principais conceitos do BOA

Entre outros, é da responsabilidade do gestor do grupo a gestão de utilizadores, a definição de uma hierarquia de tópicos (usados para categorizar os OA desse grupo), a atribuição de revisores, e a publicação de OA.

\subsection{Mecanismo de Créditos}

Uma das principais inovações do BOA, que o distinguem de outros repositórios de OA é a adopção da metáfora da "bolsa de valores" que permite traduzir facilmente o valor de cada OA (e da própria bolsa na sua totalidade) ao longo do tempo, como medida da sua utilização e popularidade.

Esta metáfora é concretizada por um mecanismo de créditos que permite atribuir 
inicialmente um determinado valor a cada $\mathrm{OA}$ e que esse valor seja actualizado periodicamente, de acordo com a sua popularidade. Possibilita ainda quantificar a colaboração dos utilizadores, não só pela criação e publicação de OA, mas também formas de colaboração como a avaliação de OA ou 0 registo de boas práticas de utilização dos OA. As diferentes formas de colaboração previstas no BOA permitem definir um ambiente de saudável competição entre os utilizadores, estimulando a colaboração entre todos os actores interessados na produção e utilização de OA. A Figura 4 sugere os dois principais elementos que estão directamente associados ao mecanismo de créditos: os OA e os utilizadores.

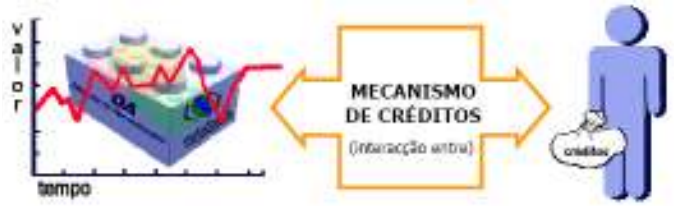

Figura 4 - Créditos atribuídos a OA e a utilizadores

O valor dos OA varia consoante o número de compras que se efectuam ao longo do tempo. Tipicamente, o utilizador ao registarse no BOA recebe uma quantidade de créditos, que é depois gasta na compra de OA existentes. Essa quantidade dispendida de créditos pode ser recuperada consoante o nível de participação e colaboração do utilizador ao associar informação relevante ao OA que acabou de adquirir, como por exemplo atribuir uma classificação ou efectuar um comentário. Salienta-se o facto de todas estas informações, associadas ao $\mathrm{OA}$, constituírem um valor acrescido e um factor determinante para os utilizadores que o pretendam adquirir.

\subsection{Mecanismo de configuração}

A versatilidade e a flexibilidade do BOA decorre da existência de mecanismos de configuração e parametrização extensa do sistema. Por um lado, pelo facto do BOA ter sido concebido e implementado sobre o sistema WebComfort, plataforma de gestão de conteúdos e aplicações Web, que oferece inúmeros aspectos de configuração e administração (SARAIVA; SILVA, 2008). Por outro lado, pelo facto do BOA ter sido concebido também com mecanismos de configuração, quer ao nível global do sistema, quer ao nível dos Grupos definidos.

A tabela da Figura 5 sugere alguns parâmetros de configuração do BOA para se adaptar a diferentes cenários de aplicação. Como exemplo, pode-se definir (1) a quantidade de créditos que cada autor recebe após a submissão de um $O A$; (2) se esse valor é determinado por uma percentagem do valor do OA ou se é um valor fixo igual para todos os OA; (3) os valores de créditos atribuídos para a colaboração dos utilizadores; e (4) a percentagem de valorização após a compra ou mesmo a desvalorização caso não tenham sido realizadas compras.

Este mecanismo de configuração permite ajustar o comportamento do sistema para se adaptar a diferentes cenários ou a possíveis alterações ao longo do tempo.

\begin{tabular}{|c|c|c|c|c|c|}
\hline & ACRÓNIMO & DESCRIÇÃo & VALOR & TIPO & TIPO DE Or \\
\hline$\theta$ & Autor & Submissão de $O A$ & 100 & $\%$ & Compra \\
\hline 0 & CompraOA & Compra de $O A$ & 100 & $\%$ & Compra \\
\hline 0 & DecrementoOA & $\begin{array}{l}\text { Actualização do valor do } O A \text { por } \\
\text { ausência de compra diária }\end{array}$ & 1 & $\%$ & Compra \\
\hline 0 & IncrementoOA & $\begin{array}{l}\text { Actualização do Valor do } \mathrm{OA} \text { por } \\
\text { cada compra diária }\end{array}$ & 1 & $\%$ & Compra \\
\hline 0 & Venda $O A$ & $\begin{array}{l}\text { Valor para os Autores por cada } \\
\text { compra de } O A\end{array}$ & 50 & $\%$ & Compra \\
\hline 0 & ClassificaOA & Classificação de um OA & 5 & VA & Submissão \\
\hline 0 & ComentarioOA & $\begin{array}{l}\text { Submissão de comentários de } \\
\text { OA }\end{array}$ & 10 & VA & Submissão \\
\hline 0 & CompraCreditos & $\begin{array}{l}\text { Compra por crédito em } \\
\text { Centimos }\end{array}$ & 10 & VA & Submissão \\
\hline 0 & RegistoUser & Registo do Utilizador & 100 & VA & Submissão \\
\hline
\end{tabular}

Figura 5 - Tabela de Configuração do BOA (visão parcial)

\subsection{Submissão de OA}

A Figura 6 sugere 0 processo de submissão do OA no sistema. O autor, para além de submeter o ficheiro do OA, regista ainda os respectivos metadados e atribui o valor inicial e o valor mínimo. A submissão do $O A$ pode ficar sujeita à análise por parte do revisor, que deve aprovar a sua publicação de acordo com a qualidade do OA e dos critérios de negócio para os valores propostos pelo autor. Caso seja aceite, o revisor efectua uma revisão que fica associada ao OA atribuindo-lhe também uma classificação, por exemplo, numa 
escala de 1 a 5. O OA fica disponível no BOA juntamente com a informação associada pelo revisor, que representa o primeiro indicador de qualidade do OA.

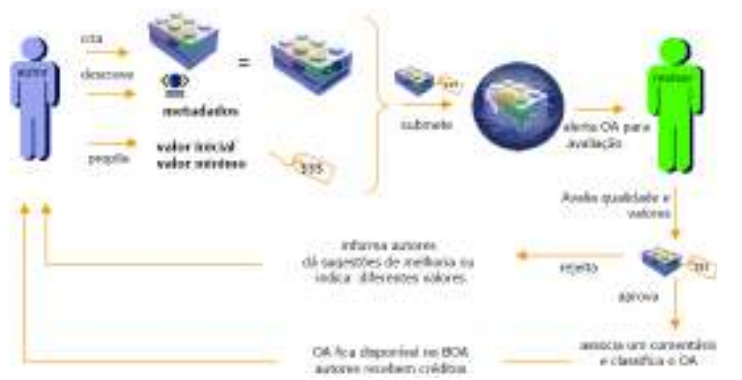

Figura 6 - Workflow de Submissão de OA

Caso o revisor rejeite o OA, deve informar o autor das razões dessa decisão, indicando sugestões de melhoria ou de reavaliação dos valores propostos. Como o valor do OA varia com o número de compras, o valor inicial é o que o OA assume no dia da sua publicação. O valor mínimo representa o limite inferior pelo qual o OA poderá ser adquirido, uma vez que a ausência de compras do OA leva a uma diminuição do seu valor. Uma vez atingido o valor mínimo, este mantém-se inalterado até que sejam efectuadas compras que possam provocar novamente a sua variação ascendente.

\subsection{Compra/Download de OA}

Existem diferentes formas de se ter acesso à página de um OA (a Figura 7 apresenta ecrã de página de um OA): por exemplo a partir dos rankings de OA em destaque ou a partir da lista de OA na página de um determinado utilizador. Todavia, a forma mais flexível é através do mecanismo de pesquisa simples ou avançada de OA. Após o resultado dessas pesquisas, é apresentada uma lista com os OA que satisfazem os critérios pretendidos.

Os utilizadores podem ver a informação associada ao OA, como os seus metadados, comentário do revisor, classificação e outro tipo de informação submetida pelos utilizadores. Caso pretendam obter o OA, necessitam apenas de possuir o número de créditos correspondente.

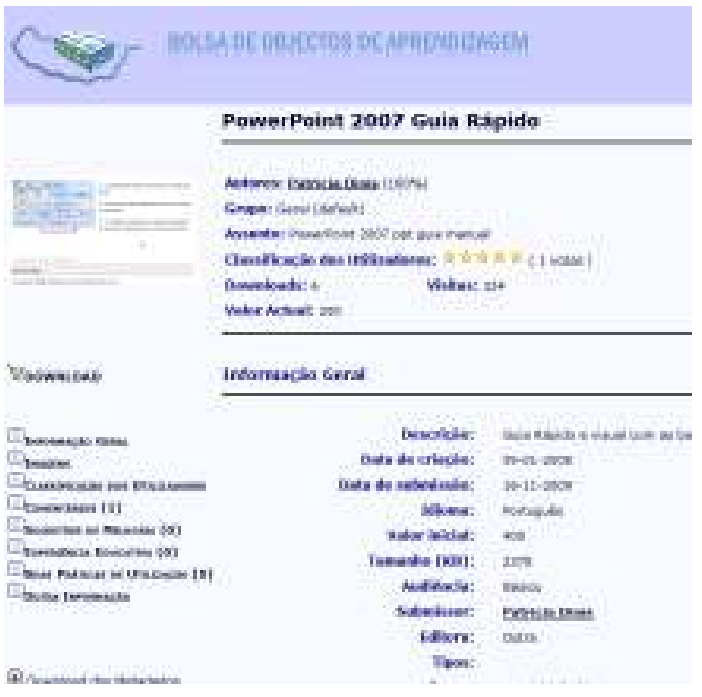

Figura 7 - Página de um OA

Após a compra do OA, o utilizador pode ainda submeter informação relevante, nomeadamente sugestões de utilização ou relato de experiências de aprendizagem. Ao submeter este tipo de informação, além de estar a complementar e a enriquecer o OA, recupera parte ou mesmo a totalidade dos créditos despendidos na compra do respectivo OA. O processo de pesquisa, compra e submissão de informação complementar está sugerido na Figura 8.

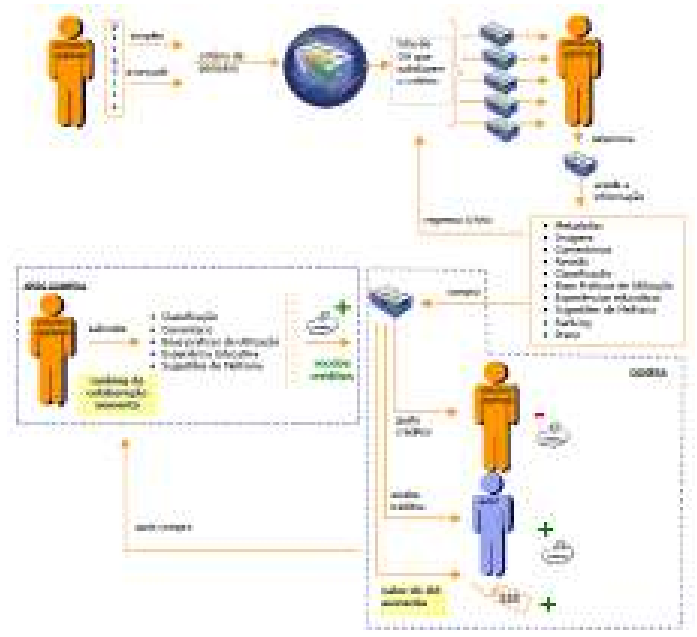

Figura 8 - Workflow de Compra de OA 


\section{Padrões de Interfaces Sociais}

No decorrer do actual trabalho de investigação, e segundo o trabalho realizado por Crumlish e Malone (2009), é identificado um conjunto de padrões de referência no desenho de interfaces sociais. São por isso adoptados neste trabalho, como ferramenta de análise e avaliação de comunidades online, os padrões de desenho social Profile, Pesrsonnaldashboard, Favorites, Displaying, Send/Share Widget, EmbBeding e Send/Receive InVITATIONS que se descrevem sucintamente de seguida.

Profile. O padrão Profile permite definir e agregar de forma adequada a informação acerca de cada utilizador no contexto da respectiva comunidade. Essa informação pessoal pode ser usada para parametrizar e personalizar a aplicação de acordo com as preferências dos seus utilizadores, pode ainda ser usada para permitir o conhecimento dos utilizadores, ou para o estabelecimento de relações sociais.

Personal Dashboard. O padrão Personal Dashboard permite que cada utilizador tenha acesso de forma fácil às principais acções que possa realizar, e ainda ao seu registo histórico de actividades na comunidade. Um utilizador pode ainda utilizar O DASHBOARD para aceder aos seus FAvorites, assim como, visualizar a que grupos ou colecções da comunidade está associado.

Favorites. O padrão Favorites permite ao utilizador marcar um objecto disponível na comunidade (seja ele uma pessoa, um lugar, um recurso, etc.) como sendo um favorito, possibilitando a criação de listas de objectos favoritos, e posteriormente o seu acesso mais rápido e flexível.

Displaying. O padrão Displaying discute as melhores formas para apresentação dos recursos à comunidade. Quando os recursos são visualizados e comentados por toda a comunidade, estão ao mesmo tempo a ser validados por todos, assim como a atribuir valor ao trabalho realizado pelo seu autor, incentivando a que novos objectos relacionados.

Send/Share Widget a Embedding. Em geral os utilizadores de comunidades online valorizam a partilha de conteúdos. Como tal, quanto mais fácil for essa acção, mais vezes a farão. Este facto apresenta um potencial para a divulgação de conteúdos dentro e fora da comunidade, sendo essas acções de partilha e divulgação em sites terceiros, ao não necessitarem da intervenção dos gestores da comunidade, são rapida e exclusivamente feitas pelos seus utilizadores, promovendo de forma viral a disseminação dos objectos e da própria comunidade.

Invitations (Send AND Receive). A aplicação do padrão INvitATIONS permite a expansão da comunidade através de um processo de marketing viral. Através de InvitATIONS, a promoção e divulgação da comunidade é feita pelos próprios utilizadores, através do envio de convites personalizados a potenciais utilizadores para que estes se juntem à comunidade e assim sucessivamente, aumentando a sua dimensão.

\section{Análise de Sistemas Baseados em Objectos Sociais}

Analisando as comunidades online existentes percebe-se porque razão umas se mantêm activas enquanto outras definham e perdem o interesse por parte dos seus utilizadores. Segundo Jyri Engeström, no seu trabalho "Building Sites Around Social Objects" (2009), isto acontece porque umas mantêm bem definido a noção central de "objecto social" enquanto outras não. Tomando como exemplo a comunidade Flickr (ver Secção 4.3), o seu objecto social principal é a fotografia, uma vez que esta é a razão principal pela qual os seus utilizadores aderem e se relacionam uns com os outros. Para além do objecto social, e ainda segundo Engeström, é igualmente importante uma comunidade online definir os seus "verbos" (ou acções). Isto significa que a comunidade deve intuir facilmente $e$ de forma simples, quais as acções que os utilizadores podem executar sobre o objecto social em causa. Assim sendo, torna-se importante na análise de comunidades online, identificar não só os seus padrões de desenho social, como também os seus Objectos Sociais e os seus Verbos. 
Nesta análise são tidas em consideração comunidades online de referência, quer em número de utilizadores quer em termos de facilidades e nível de usabilidade. Foram seleccionadas para este estudo as comunidades online Youtube, Slideshare e Flickr. Na análise destas comunidades foram usados os padrões de desenho de interfaces sociais definidos por Crumlish e Malone (2009), assim como, a identificação dos seus Objectos Sociais e Verbos, como discutido por Jyri Engeström (2009).

\subsection{Youtube}

O Youtube (www.youtube.com) é a maior comunidade online de publicação de vídeos na internet. Tem como pontos fortes um motor de busca potente (com influência do Google, universo do qual faz parte), uma interface simples e uma elevada performance de reprodução de vídeo. Permite a criação de grupos em torno de categorizações de vídeos e, ainda, de tópicos relacionados com os vídeos, por exemplo, bandas de música.

A Tabela 1 sintetiza os principais aspectos da análise considerada.

Tabela 1 - Youtube - Síntese da Análise

\begin{tabular}{|l|l|}
\hline Aspecto & Descrição \\
\hline $\begin{array}{l}\text { Objectos } \\
\text { Sociais }\end{array}$ & Vídeo \\
\hline Verbos & $\begin{array}{l}\text { play, pause, barra de } \\
\text { progresso,controlar volume, } \\
\text { gostei/não gostei, adicionar favoritos, } \\
\text { partilhar, adicionar comentário e } \\
\text { pesquisar }\end{array}$ \\
\hline $\begin{array}{l}\text { Padrões } \\
\text { Sociais } \\
\text { Suportados }\end{array}$ & $\begin{array}{l}\text { Profile, Personal Dashboard, } \\
\text { Favorites, Displaying, Send/Share }\end{array}$ \\
\hline
\end{tabular}

\subsubsection{Objectos Sociais e Verbos}

Conseguimos facilmente identificar o vídeo como sendo o Objecto Social central do Youtube. Sobre esse vídeo é perceptível através da sua interface gráfica, que as principais acções que se esperam por parte dos utilizadores são visualização, comentários, votos de gosto ou não do vídeo, adição aos favoritos, partilha do vídeo com outros utilizadores internos ou externos à comunidade e pesquisa de vídeos.

\subsubsection{Profile, Personal Dashboard e Favorites}

O Youtube permite que os utilizadores configurem o seu perfil, de modo a personalizarem a forma como acedem à informação no sistema. Entre as opções que o utilizador pode configurar, estão a informação pessoal do utilizador (dados pessoais), o formato dos vídeos ao visualizá-los (por exemplo, sempre em High Definition), se pretende partilhar vídeos através do Facebook, se pretende ter acesso a legendas nos vídeos.

A funcionalidade de PERSONAL DASHBOARD permite que o utilizador tenha acesso, após registo, a: vídeos anteriormente importados (upload) para a ferramenta; vídeos adquiridos/comprados; playlists criadas; vídeos favoritos; histórico de pesquisas e visualizações.

A funcionalidade de FAvorites permite que um utilizador marque um determinado vídeo como favorito, disponibilizando-o automaticamente na sua lista pessoal, que pode ser consultada facilmente através da opção My Videos \& Playlists acessível através da sua conta (PERSONAL DASHBOARD).

\subsubsection{Displaying}

Como se pode ver na Figura 9, o Youtube apresenta uma interface simultaneamente simples e atractiva que permite aos utilizadores acederem facilmente às principais funcionalidades da ferramenta, sendo este um dos seus principais pontos fortes.

A interface do Youtube caracteriza-se por uma barra localizada na lateral direita, na qual surgem as sugestões feitas pelo sistema, com base no que o utilizador e outros utilizadores em buscas semelhantes, visualizaram anteriormente. No topo e de forma destacada, surge a barra de pesquisa; à sua direita, o acesso aos dados pessoais do utilizador e, na zona central, e dependendo do contexto, surgem os resultados da pesquisa feita pelo utilizador 
ou, em alternativa, o reprodutor com o vídeo que o utilizador seleccionou. O reprodutor apresenta comandos simples apenas com as funcionalidades necessárias para controlar a reprodução de vídeos, nomeadamente, play, pause, barra de progresso, maximizar imagem, restaurar imagem e controlar volume.

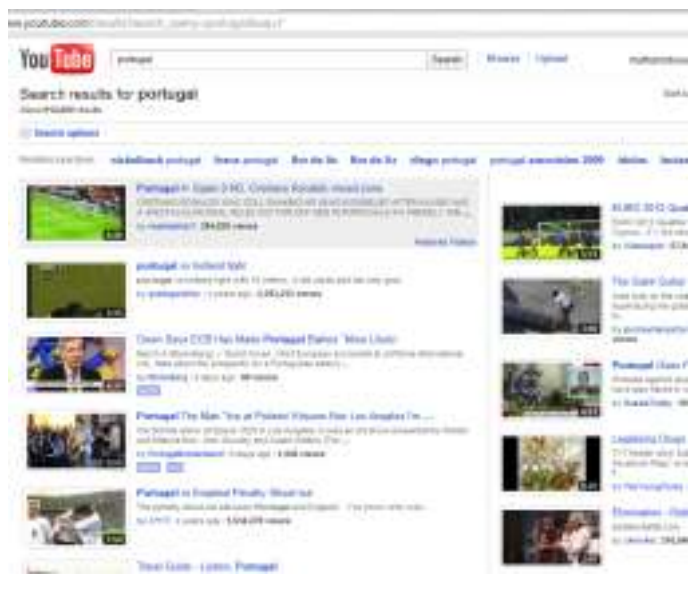

Figura 9 - Aspecto da interface do Youtube

\subsubsection{Send/Share Widget e Embedding}

Uma das funcionalidades mais usadas pelos utilizadores do Youtube, e presente em milhões de sites, refere-se à possibilidade de integrar ou referenciar nesses sites os vídeos mantidos no Youtube, permitindo, por exemplo, que um utilizador do sistema Facebook comente no seu perfil um determinado vídeo publicado no Youtube. É ainda possível, recorrendo à funcionalidade de EMBEDDING, publicar num site terceiro, um vídeo nele alojado, aumentando desta forma o número de utilizadores que the acedem. Estas formas de interacção promovem uma dinâmica entre utilizadores sem qualquer intervenção quer dos gestores do Youtube, quer dos gestores de sites externos a este.

\subsubsection{Send/Receive Invitations}

No Youtube não existe a lógica de envio de invitations como forma de aumentar o número de utilizadores. O registo no sistema é livre e sem restrições, sendo os novos utilizadores recrutados através de mecanismos de marketing "boca-a-boca" dos utilizadores já registados, publicação e partilha de vídeos em sites externos. Apenas após o registo do utilizador no sistema, podem ser enviados convites para pertencer a um determinado grupo ou comunidade, ou ainda o envio de mensagens pessoais entre utilizadores.

\subsection{SlideShare}

O SlideShare (www.slideshare.net) é um sistema que permite aos seus utilizadores, publicarem e partilharem as suas apresentações e documentos pessoais e/ou profissionais. Este sistema permite que milhares de utilizadores façam upload de ficheiros nos formatos PPT, PPS, PPTX, ODP, PDF, DOC, DOCX, ODT, Keynote e iWork, assim como vídeos do Youtube e ficheiros de áudio, que podem ser associados às apresentações.

A Tabela 2 sintetiza os principais aspectos da análise considerada.

Tabela 2 - SlideShare - Síntese da Análise

\begin{tabular}{|l|l|}
\hline Aspecto & Descrição \\
\hline $\begin{array}{l}\text { Objecto } \\
\text { Social }\end{array}$ & Apresentação \\
\hline Verbos & $\begin{array}{l}\text { visualizar, upload, download, } \\
\text { pesquisar, partilhar e adicionar } \\
\text { favoritos }\end{array}$ \\
\hline $\begin{array}{l}\text { Padrões } \\
\text { Sociais } \\
\text { Suportados }\end{array}$ & $\begin{array}{l}\text { Profile, Personal Dashboard, } \\
\text { Favorites, Displaying, Send/Share } \\
\text { widget, Embedding, Send/Receive } \\
\text { Invitations }\end{array}$ \\
\hline
\end{tabular}

\subsubsection{Objecto Social e Verbos}

No caso do Slideshare, o Objecto Social identificado é a apresentação (seja em que formato for). Os utilizadores da comunidade têm como principais acções disponíveis: pesquisar, visualizar, partilhar, adicionar aos favoritos, fazer upload, fazer download das apresentações.

\subsubsection{Profile, Personal Dashboard e Favorites}

A personalização do perfil do utilizador no Slideshare, permite que este tenha acesso a: informação do seu histórico de 
participação na comunidade; receber newsletters às quais se tenha associado; definir o seu perfil público (visível pelos outros utilizadores da comunidade); listagem de todos os seus uploads; informação pessoal detalhada; acesso a dados estatísticos sobre consultas às publicações de que é autor.

\subsubsection{Displaying}

A interface gráfica do Slideshare (Figura 10) é caracterizada por uma barra na lateral direita, com informação relativa à apresentação do autor: nome, descritivo, outras apresentações do mesmo autor e uma listagem de outras apresentações a que esta está associada e que servem de possível sugestão ao utilizador. No topo encontramos uma barra com o motor de pesquisa, as opções de configuração do perfil do utilizador e as ferramentas de envio da apresentação por email, adição aos favoritos, download. $\mathrm{Na}$ barra lateral esquerda o utilizador encontra ferramentas de partilha da apresentação noutras comunidades (ex: twitter, facebook). Na zona central encontramos o visualizador da apresentação com comandos simples de reprodução como play, pause, forward, backward, início, fim, maximizar e restaurar imagem. Na zona abaixo do visualizador, o utilizador encontrará a listagem de todos os comentários deixados acerca da apresentação exibida no visualizador.

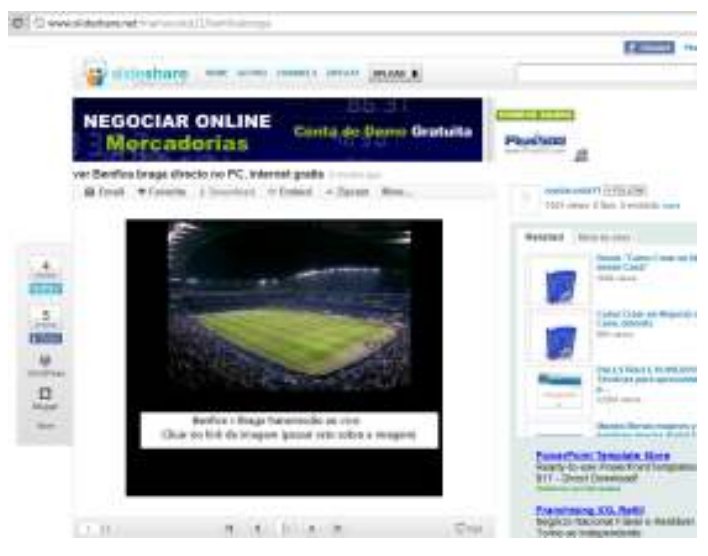

Figura 10 - Aspecto da interface do Slideshare

\subsubsection{Send/Share Widget e Embedding}

É possível partilhar um objecto (publicação) do Slideshare, com outros utilizadores/pessoas de diversas formas. Desde logo, através da integração entre o Slideshare e o Facebook, Twitter, WorldPress, o Blogger, e outras comunidades. É também possível a partilha de publicações através de email, enviandose ao destinatário o URL permanente onde se encontra a apresentação ou ainda através da disponibilização do código HTML da apresentação, de forma a colocá-la num outro qualquer site na internet.

\subsubsection{Send/Receive Invitations}

O serviço de convites, no Slideshare, à semelhança de outros, funciona como divulgador da comunidade e incentivador à sua expansão em termos de número de utilizadores. É possível a um utilizador convidar amigos para se juntarem a si no Slideshare, através do envio selectivo de convites para o email desses seus amigos ou através da importação dos seus contactos a partir da sua conta de email (possível apenas a partir dos servidores mais populares mail - Yahoo, Gmail, Hotmail).

\subsection{Flickr}

O Flickr (www.flickr.com) é uma das mais populares comunidades online, que se dedica à publicação de fotografias disponibilizadas pelos seus utilizadores. Esta comunidade permite que os seus utilizadores divulguem as suas galerias (fotografias) na internet, disponibilizando ainda, funcionalidades de criação de grupos, rankings de fotos por tópicos, mapa com registo geográfico do local onde a fotografia foi tirada, entre outras.

A Tabela 3 sintetiza os principais aspectos da análise considerada. 
Tabela 3 - Flickr - Síntese da Análise

\begin{tabular}{|l|l|}
\hline Aspecto & Descrição \\
\hline $\begin{array}{l}\text { Objecto } \\
\text { Social }\end{array}$ & Fotografia \\
\hline Verbos & $\begin{array}{l}\text { Publicar, adicionar favoritos, partilhar } \\
\text { e pesquisar }\end{array}$ \\
\hline $\begin{array}{l}\text { Padrões } \\
\text { Sociais } \\
\text { Suportados }\end{array}$ & $\begin{array}{l}\text { Profile, Personal Dashboard, } \\
\text { Favorites, Displaying, Send/Share } \\
\text { widget, Embedding, Send/Receive } \\
\text { Invitations }\end{array}$ \\
\hline
\end{tabular}

\subsubsection{Objectos Sociais e Verbos}

Na comunidade Flickr o Objecto Social é facilmente identificável como sendo a fotografia, sendo o atractor principal desta comunidade. Entre as principais acções que os utilizadores podem efectuar, para além da publicação de fotografias, identifica-se a possibilidade de as adicionar aos favoritos, comentar, visualizá-las em diferentes formatos, partilhá-las com outros utilizadores e pesquisá-las.

\subsubsection{Profile, Personal Dashboard e Favorites}

A personalização na comunidade Flickr, permite que o utilizador tenha acesso à sua galeria privada, às suas fotografias favoritas, ao seu histórico de actividades, fazer upload de fotografias, aceder aos seus dados pessoais detalhados, ter acesso à sua conta de email dentro da comunidade, ter acesso à funcionalidade de mapa, que lhe permite localizar geograficamente os locais onde as suas fotografias foram tiradas. Outra das funcionalidades interessantes que a personalização permite, passa pela organização pessoal de todos os álbuns. É ainda possível, ao nível do perfil do utilizador, gerir a sua lista de contactos e amigos, com os quais interage ou interagiu (através do respectivo histórico), assim como, associar blogs ao seu perfil, de forma a poder interagir com estes, de forma automática.

\subsubsection{Displaying}

Como é possível ver pela Figura 11, o Flickr apresenta uma interface simples. A barra no topo permite efectuar pesquisas, configurar as opções do perfil de utilizador, adicionar a fotografia seleccionada aos seus favoritos, visualizá-la em diferentes modos (escolher o tamanho, ver em slideshow, ver informação técnica detalhada da fotografia Exif), enviar a fotografia a um amigo via email e visualizar outras fotos do mesmo autor (opções mais recente e mais antigo). $\mathrm{Na}$ barra lateral direita o utilizador tem acesso a informação resumida do autor e uma breve descrição da fotografia seleccionada, assim como, a uma listagem das fotos do mesmo autor (galeria) e dos grupos a que a fotografia está associada, apresentadas como forma de sugestão ao utilizador. No centro do ecrã surge a fotografia seleccionada pelo utilizador, com a opção de passar ao modo expandido clicando sobre a imagem. É ainda possível ter acesso a comentários deixados por outros utilizadores, a zonas específicas da imagem, bastando para isso parar o rato sobre essas mesmas zonas da imagem (identificadas por pequenos quadrados que contornam essas mesmas zonas). Na zona inferior do ecrã, o utilizador encontra os comentários feitos por outros utilizadores à fotografia seleccionada, notando-se a particularidade de surgir, junto ao comentário, a referência de que essa fotografia foi adicionada como favorita pelo autor desse comentário. No final desta, surge então a opção de também o utilizador deixar um comentário à fotografia seleccionada.

\subsubsection{Send/Share Widget e Embedding}

É possível a partilha de fotografias no Flickr, através de várias estruturas disponibilizados por esta comunidade. Esta partilha é possível através de um mecanismo de envio da fotografia por email para um qualquer contacto escolhido pelo utilizador. Também é possível partilhar estes objectos, através da geração de URL (link) permanente, gerado e facultado automaticamente pelo Flickr. É ainda possível obter o respectivo código (quer seja em HTML ou em BBCode) que permite embeber uma fotografia publicada no Flickr, 
num qualquer site da internet. Finalmente, é ainda possível partilhar uma fotografia do Flickr num qualquer blog de forma automática, através de um mecanismo, disponibilizado pelo Flickr, que recorre à configuração do perfil do utilizador, associando-lhe blogs.

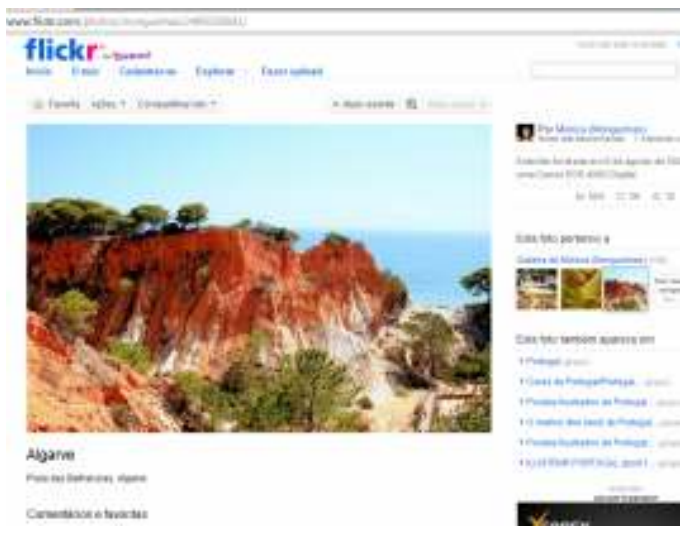

Figura 11 - Aspecto da interface do Flickr

\subsubsection{Send/Receive Invitations}

Através do sistema de envio de convites do Flickr, é possível adicionar a esta comunidade outros utilizadores, tais como amigos ou família. É também possível, à semelhança de outras comunidades, importar contactos de outros utilizadores, através do recurso à importação de contas de email dos servidores mais populares (Yahoo, Gmail, Hotmail e Facebook). Com este mecanismo pretende-se que a comunidade se expanda de forma dinâmica e automática, com a vantagem de serem os utilizadores os únicos intervenientes neste processo, o que apresenta por si só benefícios quer para a comunidade quer para os responsáveis pela sua gestão.

\section{Discussão}

Utilizando como base a teoria dos sistemas centrados em objectos sociais e a análise de comunidades online como o Youtube, Slideshare e Flickr (ver Secção 4), discute-se nesta secção a versão actual do BOA com o objectivo de identificar e propôr alterações que mitiguem as suas limitações actuais. A versão da plataforma BOA considerada está disponível em www.vemaprender.net e corresponde à versão disponível ao público de registo e utilização livre.

A Tabela 4 sintetiza os principais aspectos da análise considerada à versão actual do BOA.

Tabela 4 - BOA - Síntese da Análise Actual

\begin{tabular}{|l|l|}
\hline Aspecto & Descrição \\
\hline $\begin{array}{l}\text { Objecto } \\
\text { Social }\end{array}$ & Objecto de Aprendizagem \\
\hline Verbos & $\begin{array}{l}\text { Submeter, criar colecção, } \\
\text { pesquisar, consultar, } \\
\text { download/comprar, comentar, } \\
\text { classificar, rever }\end{array}$ \\
\hline $\begin{array}{l}\text { Padrões } \\
\text { Sociais } \\
\text { Suportados }\end{array}$ & $\begin{array}{l}\text { Profile, Personal Dashboard, } \\
\text { Displaying }\end{array}$ \\
\hline
\end{tabular}

\subsection{Objectos Sociais e Verbos}

Tal como acontece nas comunidades analisadas, o Objecto Social e os Verbos do BOA estão bem definidos. O objecto social do BOA é o "Objecto de Aprendizagem", ou seja, o recurso digital que pode ser partilhado e reutilizado como suporte a diferentes processos e cenários de ensinoaprendizagem. Relativamente aos verbos estes encontram-se também bem definidos, estando essencialmente disponíveis na secção "Principal" (ver Figura 2) e associadas ao próprio objecto na sua página de detalhe. As principais acções são: Submeter OA, Criar colecção de OA, Pesquisar OA, Consultar OA, Download de OA, Avaliar OA, Submeter Comentários, Experiências Educativas, Sugestões ou Boas Práticas de Utilização.

\subsection{Profile, Personal Dashboard e Favorites}

Em termos de definições pessoais do utilizador, este pode configurar os seus dados pessoais tais como o nome, morada, email, telefone, fotografia. O utilizador tem a possibilidade de personalizar o seu perfil com a inclusão de uma fotografia, assim como escolher qual o seu tipo de utilizador (e.g., aluno, docente, encarregado de educação ou outro), a escola a que 
pertence, e ainda através de que meio teve conhecimento da comunidade BOA. A página pessoal (ver Figura 12) de cada utilizador apresenta o seu historial de actividade, e também um gráfico com a variação dos créditos que o utilizador vai adquirindo $\mathrm{e}$ dispendendo ao longo do tempo, a lista dos objectos que é autor e ainda a lista com as operações que realizou (e.g., compras, vendas, comentários adicionados).

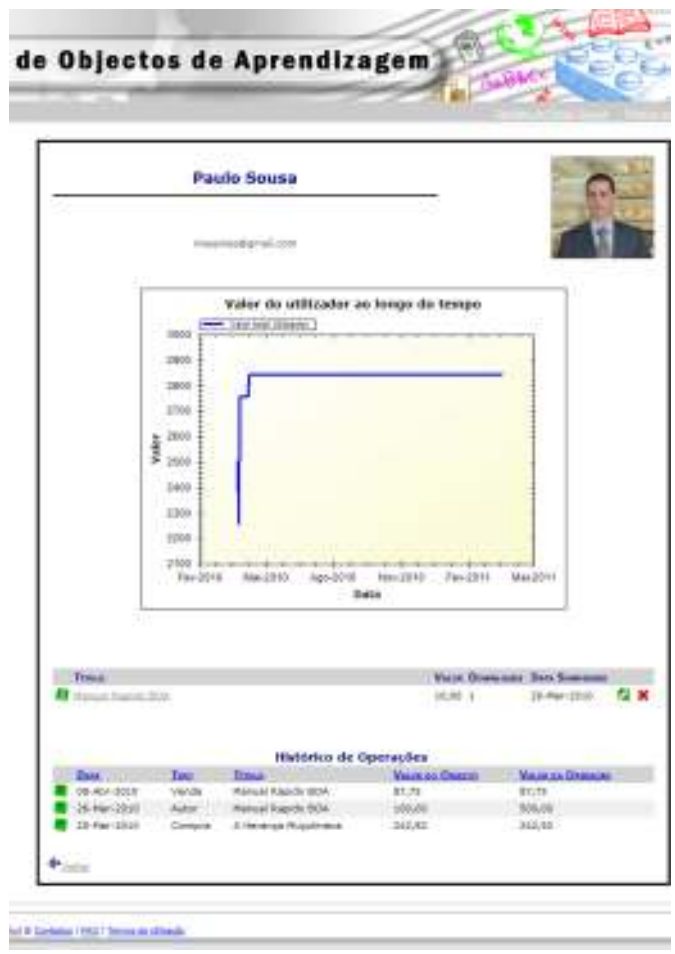

Figura 12 - Página de perfil de utilizador no BOA

Em termos de comparação com as comunidades analisadas, o BOA segue as boas práticas, permitindo ao seu utilizador configurar o seu perfil. Possibilita o acesso ao perfil dos restantes utilizadores, permitindo o estabelecimento de relações entre eles, assim como, conhecer melhor os autores dos OA consultados e vice-versa.

\subsection{Displaying}

O BOA apresenta uma interface caracterizada por uma barra no topo que permite 0 acesso às opções do perfil do utilizador (ver Figura 6). Permite aceder a cada uma das secções da comunidade através de separadores/tabs. Na barra lateral esquerda surgem as opções que dependem sempre do contexto em que o utilizador se encontra. Na página principal as opções consistem em atalhos para acções que o utilizador executa com mais frequência, tais como: consultar a página pessoal, submeter objectos, criar colecções de objectos, pesquisar e acesso a informação de resumo das estatísticas gerais de actividade da comunidade. No centro do ecrã surge a informação que o utilizador pretende ver, dependendo do contexto (por exemplo, no caso de uma pesquisa, surge a lista de OA seleccionados).

Como meio de pré-visualização dos OA, e quando comparado com outras comunidades, deparamo-nos com uma relevante limitação no BOA. A única forma que um utilizador tem para ter acesso a uma pré-visualização de um Objecto de Aprendizagem, antes de fazer o respectivo download, é se este tiver associado imagens, acedendo a estas (como se pode ver na Figura 13). No caso do submissor não adicionar nenhuma imagem do seu objecto, os restantes utilizadores apenas conseguirão ter acesso a ele, após fazerem o respectivo download, o que de certa forma constitui uma barreira a que isso mesmo aconteça.

Uma forma de amenizar esta limitação é criar mecanismos de pré-visualização de segmentos do objecto, por exemplo, dando aos utilizadores, o acesso a uma amostra do objecto. Este mecanismo tem de ser dependente do formato de dados do OA (e.g., DOC, PPT, PDF, MPEG). 


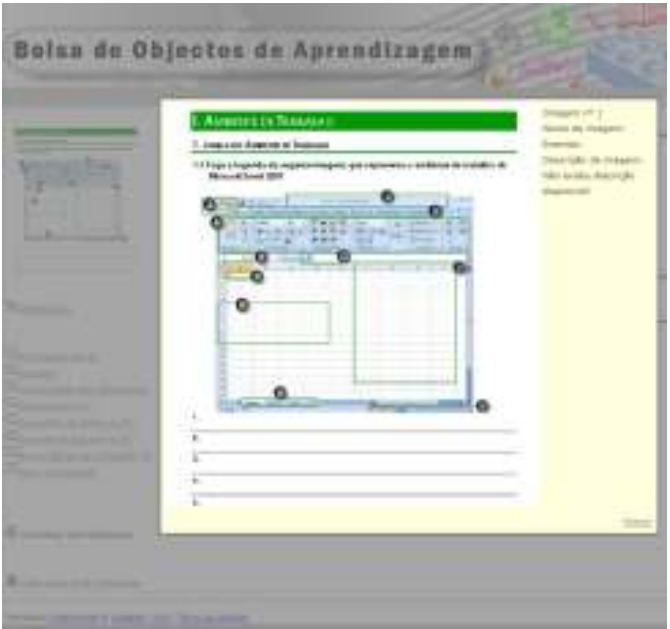

Figura 13 - Aspecto do pré-visualizador de imagens

A mitigação desta limitação ao nível de apresentação dos OA e da pré-visualização dos seus conteúdos pode conduzir a maior adesão e interesse dos utilizadores, em particular ao potenciar a descoberta de OA adequados e consequente download.

\subsection{Send/Share Widget e Embedding}

Analisando o padrão Send/SHARE Widgets e embedding, ao contrário das outras comunidades analisadas, o BOA não apresenta opções, estando apenas limitado à disponibilização de um serviço de RSS com a indicação dos últimos OA adicionados. Como se observou nas análises anteriores, estes mecanismos constituem uma aposta significativa para a divulgação não só dos objectos mas também, indirectamente, da própria comunidade, permitindo que sejam os utilizadores os seus principais divulgadores.

Uma das técnicas de atenuar esta limitação é, por exemplo, recorrer à geração de código HTML (embedded code) que permita embutir em sites externos (e.g., Facebook, blogs pessoais) referências para os OA alojados no BOA, tornando desta forma o BOA mais conhecido através da sua ligação a sites terceiros.

\subsection{Send/Receive Invitations}

A actual versão do BOA não suporta a funcionalidade correspondente ao padrão Send/Receive invitations. Sendo este padrão distintivo para a divulgação e a promoção de comunidades online através do modelo de marketing viral, considera-se fundamental o seu desenvolvimento no âmbito do presente trabalho de investigação. Considera-se ainda que este mecanismo deverá ser complementado através de um mecanismo de incentivos (e.g., concretizados pela concessão de créditos) a atribuir directamente aos utilizadores que enviem convites pelos seus contactos.

\section{Conclusão}

O BOA consiste num sistema de suporte a comunidades online centradas em processos de ensino-aprendizagem. Como qualquer comunidade, presupõe uma colaboração activa por parte dos seus utilizadores (PREECE; MALONEY-KRICHMAR, 2003), (IRIBERRI; LEROY, 2009), neste caso, através da produção e submissão de OA de qualidade, mas também pela submissão de informação adicional que auxilie na compreensão ou utilização mais eficaz desses mesmos OA, e.g. através da submissão de comentários, partilha de experiências educativas, boas práticas de utilização, ou sugestões de melhoria.

Como sugerido no artigo "Casos de Estudo com a Bolsa de Objectos de Aprendizagem: Análise na Perspectiva da Computação Social" (SILVA; DINIS, 2010), e discutido nesta Secção 5, embora o BOA siga grande parte dos padrões de desenho de interfaces sociais de comunidades online, há ainda um espaço de crítica e melhoria a realizar. Este artigo focou-se nas facetas sócio-técnicas que são alvo de investigação e desenvolvimento. (As facetas de carácter organizacional e social, ainda que decisivas, saiem deliberadamente fora do âmbito da discussão deste artigo (IRIBERRI; LEROY, 2009), (WASKO, 2005).)

Do resultado da análise apresentada realça-se a necessidade de melhorar funcionalidades já existentes e mesmo de 
introduzir novas funcionalidades. A Tabela 5 sintetiza os principais aspectos que se consideram mais significativos desenvolver no âmbito do actual trabalho de investigação.

Tabela 5 - BOA - Síntese dos aspectos em desenvolvimento

\begin{tabular}{|c|c|}
\hline Aspecto & Descrição \\
\hline $\begin{array}{l}\text { Objecto } \\
\text { Social }\end{array}$ & Objecto de Aprendizagem \\
\hline Verbos & $\begin{array}{l}\text { submeter, criar colecção, } \\
\text { pesquisar, consultar, } \\
\text { download/comprar, } \\
\text { comentar, classificar, rever }\end{array}$ \\
\hline $\begin{array}{l}\text { Novos } \\
\text { Verbos }\end{array}$ & $\begin{array}{l}\text { adicionar OA, Autor e Grupo aos } \\
\text { Favoritos, } \\
\text { pré-visualizar OA, } \\
\text { pesquisar Autor } \\
\text { partilhar OA } \\
\text { convidar }\end{array}$ \\
\hline $\begin{array}{l}\text { Padrões } \\
\text { Sociais a } \\
\text { Melhorar e } \\
\text { ou } \\
\text { Desenvolver }\end{array}$ & $\begin{array}{l}\text { Profile } \\
\text { Personal Dashboard, Favorites } \\
\text { Displaying } \\
\text { Send/Share widget } \\
\text { Embedding } \\
\text { Invitations }\end{array}$ \\
\hline
\end{tabular}

Relativamente ao padrão Profile deverá ser possível a definição mais rica do perfil de utilizador, e.g., indicar o nível de ensino e os tópicos de interesse pretendidos na definição de preferências para, consequentemente, uma pesquisa de OA com maior eficácia. Também deverá ser permitido a pesquisa de autores para facilitar, uma vez mais, a pesquisa de OA relevantes, a partir das páginas dos respectivos autores.

O Personal Dashboard de cada utilizador, para além de apresentar a lista das suas actividades realizadas na comunidade, deverá apresentar de forma simples a lista dos OA adquiridos, mas também listas de favoritos de OA, de Autores e de Grupos de forma a acelerar e simplificar o acesso a esses recursos, e segundo a aplicação do padrão FAVorItes.
Relativamente ao padrão DISPLAYING serão acrescentadas melhorias que permitam que os OA possam ser consultados sob forma de amostra, antes de se fazer o respectivo download. Tipicamente deverão ser providenciados mecanismos de pré-visualização para os principais tipos de ficheiros (e.g., DOC, PPT, PDF, MPEG). Dando como exemplo um OA com um ficheiro no formato PDF, este será disponibilizado de forma embutida na própria página, mostrando apenas as primeiras páginas. Por outro lado, caso o formato dos dados seja vídeo, deverá ser permitido visualizar apenas um snapshot do respectivo vídeo.

Quanto às melhorias a serem implementadas em termos dos padrões Send/Share widget e Embedding, é fundamental assegurar a disponibilização, para cada $\mathrm{OA}$, do respectivo código $\mathrm{HTML}$, que permita que qualquer utilizador possa partilhar a sua referência e descrição em qualquer site externo. À semelhança do que acontece com as comunidades analisadas neste artigo, este tipo de funcionalidade potencia uma dinâmica na comunidade, pelo facto dos OA potencialmente ficarem mais expostos a mais utilizadores.

Relativamente ao padrão INvitATIONS, pretende-se desenvolver um mecanismo que permita o envio personalizado de convites para endereços de email que os utilizadores designem. Será definido um mecanismo de incentivos/bónus com atribuição de créditos aos utilizadores que realizem esses convites. Desta forma o utilizador dará a conhecer aos seus contactos a existência do BOA, permitindo que a comunidade cresça e se dissemine de forma viral pelo maior número possível de utilizadores.

Finalmente, há que referir um trabalho em aberto de integração do BOA com outras plataformas de objectos de aprendizagem, em particular enquadrado nos esforços internacionais de interoperação de objectos de aprendizagem promovidos, entre outros, pela GLOBE (Global Learning Objects Brokered Exchange, www.globe-info.org) ou a LACLO (Latin American Community of Learning Objects, www.laclo.org). 


\section{Agradecimentos}

Agradecimento a todos os investigadores que participaram directa ou indirectamente no projecto "Bolsa de Objectos de Aprendizagem", em particular ao Paulo Sousa (que contribuíu numa versão preliminar deste artigo), ao João Carlota e à Patrícia Dinis que realizaram as suas dissertações de mestrado neste âmbito. Esta investigação foi realizada com apoio FCT (INESCID multi-annual funding) e do CYTED SOLITE (Rede de cooperação Ibero-Americana para a área das tecnologias da área do ensino-aprendizagem).

\section{Referências}

CARLOTA, J., SILVA, A.R., AND DINIS, P., "The Learning Object Pool and the BOA-GPI Case Study", in Proceedings of the Iberoamerican Congress on Telematics (CITA-2009), May 2009.

CRUMLISH, C.; MALONE, E., Designing Social Interfaces, O'Reilly Media, 2009.

DINIS, P. AND SILVA, A.R. Application Scenarios for the Learning Objects Pool. Journal of Universal Computer Science, Vol. 15, No. 7, 2009.

DOORTON, M., ET AL., Transforming existing contents into reusable learning objects, in Online education using learning objects. 2004, RoutledgeFalmer.

DOWNES, S. Learning Objects - Resources for learning worldwide, in Online education using learning objects, R. Macgreal, Editor. 2004, RoutledgeFalmer.

ENGESTROM, J. Building Sites Around Social Objects - Web 2.0. Expo SF 2009.

http://www.slideshare.net/jyri/building-sites-around-social-objects-web-20-expo-sf-2009

IRIBERRI, A. AND LEROY, G., A Life-Cycle Perspective on Online Community Success. ACM Computer Surveys, Vol.41(2), Feb, 2009.

MCGREAL, R., Online education using learning objects. Open and flexible learning series. 2004, RoutledgeFalmer.

MERLOT. Multimedia Educational Resource for Learning and Online Teaching. http://www.merlot.org.

PREECE, J. E MALONEY-KRICHMAR, D., Online Communities. In Handbook of Human-Computer Interaction, Lawrence Erlbaum Associates, 2003.

SARAIVA, J.; SILVA, R.S. The WebComfort Framework: an Extensible Platform for the Development of Web Applications, in Proceedings of the 34th EUROMICRO Conference on Software Engineering and Advanced Applications, Service and Component-Based Software Engineering Track, IEEE Computer Society, 2008.

SILVA, A.R.; DINIS, P. Casos de Estudo com a Bolsa de Objectos de Aprendizagem: Análise na Perspectiva da Computação Social, In Proceedings of IE'2010 Conference (Congreso Iberoamericano de Informática Educativa), 2010.

SILVA, P.; SILVA, A.R. Análise Funcional de Plataformas de Objectos de Aprendizagem, in Proceedings of the 6th Iberoamerican Congress on Telematics (CITA-2006), May2006.

WASKO, M. Why Should I Share? Examining Social Capital and Knowledge Contribution in Electronic Networks of Practice. MIS Quarterly, Vol.29(1), March, 2005.

Recebido em maio de 2011

Aprovado para publicação em junho de 2011

\section{Alberto Rodrigues da Silva}

Professor do Departamento de Engenharia Informática do Instituto Superior Técnico da Universidade Técnica de Lisboa, Sócio e Director da empresa SIQuant e Investigador Sénior do INESC-ID, Lisboa Portugal. E-mail: alberto.silva@acm.org 